\title{
Impact of recipient-related factors on structural dysfunction of xenoaortic bioprosthetic heart valves
}

\section{Olga Barbarash \\ Natalya Rutkovskaya \\ Oksana Hryachkova \\ Olga Gruzdeva \\ Evgenya Uchasova \\ Anastasia Ponasenko \\ Natalya Kondyukova \\ Yuri Odarenko \\ Leonid Barbarash}

Federal State Budgetary Scientific Institution Research Institute for Complex Issues of Cardiovascular Diseases, Kemerovo, Russia
Correspondence: Evgenya Uchasova Federal State Budgetary Scientific Institution Research Institute for Complex Issues of Cardiovascular Diseases, 6 Sosnovy Boulevard, Kemerovo 650002, Russia $\mathrm{Tel}+73842640553$

Email evg.uchasova@yandex.ru
This article was published in the following Dove Press journal:

Patient Preference and Adherence

9 March 2015

Number of times this article has been viewed

Objective: To analyze the influence of recipient-related metabolic factors on the rate of structural dysfunction caused by the calcification of xenoaortic bioprostheses.

Materials and methods: We retrospectively analyzed clinical status, calcium-phosphorus metabolism, and nonspecific markers of inflammatory response in bioprosthetic mitral valve recipients with calcific degeneration confirmed by histological and electron microscopic studies (group 1, n=22), and in those without degeneration (group 2, n=48).

Results: Patients with confirmed calcification of bioprostheses were more likely to have a severe clinical state (functional class IV in $36 \%$ in group 1 versus $15 \%$ in group $2, P=0.03$ ) and a longer cardiopulmonary bypass period (112.8 \pm 18.8 minutes in group 1 versus $97.2 \pm 23.6$ minutes in group $2, P=0.02$ ) during primary surgery. Patients in group 1 demonstrated moderate hypovitaminosis D (median 34.0, interquartile range [21.0; 49.4] vs 40 [27.2; 54.0] pmol/L, $P>0.05)$, osteoprotegerin deficiency $(82.5[44.2 ; 115.4]$ vs $113.5[65.7 ; 191.3] \mathrm{pg} / \mathrm{mL}, P>0.05)$ and osteopontin deficiency $(4.5[3.3 ; 7.7]$ vs $5.2[4.1 ; 7.2] \mathrm{ng} / \mathrm{mL}, P>0.05)$, and significantly reduced bone-specific alkaline phosphatase isoenzyme $(17.1[12.2 ; 21.4]$ vs $22.3[15.5 ; 30.5]$ $\mathrm{U} / \mathrm{L}, P=0.01)$ and interleukin-8 levels $(9.74[9.19 ; 10.09] \mathrm{pg} / \mathrm{mL}$ vs $13.17[9.72 ; 23.1] \mathrm{pg} / \mathrm{mL}$, $P=0.045$ ) compared with group 2 , with an overall increase in serum levels of proinflammatory markers.

Conclusion: Possible predictors of the rate of calcific degeneration of bioprostheses include the degree of decompensated heart failure, the duration and invasiveness of surgery, and the characteristics of calcium-phosphorus homeostasis in the recipient, defined by bone resorption and local and systemic inflammation. The results confirm the hypothesis that cell-mediated regulation of pathological calcification is caused by dysregulation of metabolic processes, which are in turn controlled by proinflammatory signals.

Keywords: bioprostheses, calcium-phosphorus metabolism, inflammation, calcification

\section{Background}

The life span of bioprosthetic (BP) heart valves limits their application in patients undergoing surgeries for congenital and acquired heart diseases. ${ }^{1-4}$ Calcification of xenomaterials in BP heart valves is a common underlying cause of symptomatic dysfunction, leading to severe disturbances in intracardiac hemodynamics and the need for reoperation. . $^{1,3,5,6}$

The pathological calcification of soft tissues is a complex multifactorial process controlled at genetic and biochemical levels. ${ }^{7-10}$ Moreover, the calcific degeneration rate of BPs is related to the characteristics of the implanted device, as well as the metabolic status of the recipient. ${ }^{11-13}$ However, the detailed underlying mechanisms affecting BP heart-valve calcification have not yet been studied, and calcification of 
chemically modified biomaterials remains a problem, despite improvements in BPs and the use of different methods of anticalcification.

The current study investigated the effects of metabolic factors in terms of calcium-phosphorus homeostasis and proinflammatory status in recipients of BP heart valves on the rate of structural dysfunction provoked by the calcification of biomaterials.

\section{Materials and methods}

Epoxy-treated xenoaortic and xenopericardial BPs, along with mechanical valves, have been used to repair heart defects at the Research Institute for Complex Issues of Cardiovascular Diseases (NII KPSSZ, Kemerovo, Russia) for almost a quarter of a century. This experience has allowed us to evaluate the long-term outcomes of BPs in relation to specific recipient-related metabolic factors that may act as potential predictors of calcific degeneration.

Considering the differing effects of xenogenic tissue characteristics, preservation method, and mechanical load during the cardiac cycle on the rate of pathological calcification, the present study was limited to recipients with epoxy-treated valves fabricated from aortic porcine complex, implanted in the mitral position. ${ }^{5,14}$ Patients with multivalve replacements were included in the study if a mitral BP was implanted.

Monitoring of the outcomes after clinical application of a xenoaortic BP was performed using a specially designed database included in the Medical Information System of NII KPSSZ. The patients were registered after their first visit to the clinic and the following data were recorded: personal data, main diagnosis and comorbidities, echocardiography findings before and after surgical repair, data on the implanted device, and perioperative and in-hospital complications. The database also recorded information on long-term postoperative dynamic patient monitoring and on the timing and causes of BP dysfunction, as confirmed by histology and electron microscopy of the explanted valves.

A total of 382 xenoaortic BPs were implanted in the mitral position from 1991 to 2009. The surgeries were performed according to the standard procedure for mitral valve replacement under normothermic cardiopulmonary bypass (CPB). The in-hospital mortality was $5.8 \%(n=22)$, and 360 patients with BPs were successfully discharged from hospital. All the patients underwent regular follow-up visits (one visit per 1-2 years), which included a general examination and transthoracic echocardiography to assess the morphological function of the implanted BPs, and to adjust medical therapy if necessary. A total of $94.5 \%$ of patients completed the follow-up period (mean follow-up period $8.0 \pm 4.4$ years), for a total of 2,792 patient-years. The longterm mortality, including reoperations, was $29.2 \%(n=105)$ of all discharged patients. The present study included recipients of models of the epoxy-treated xenoaortic prostheses KemKor and PeriCor (Neokor, Russia).

In December 2013, 113 patients (31.4\% of all discharged patients) had undergone reoperation as a result of BP dysfunction. The causes of BP dysfunction were identified in 105 (93\%) cases (Table 1), based on histological and electron microscopic studies. The most common cause of BP dysfunction was primary tissue degeneration with calcification (46.9\%) (Figures 1-5). Prosthetic valve endocarditis occurred in $25.7 \%$ of cases (Figures 6-8). Structural degeneration caused by leaflet disruption without calcification and/or infection occurred in $12.4 \%$ of cases. Calcium deposits (crystalline and amorphous forms), as well as leukocytic/lymphocytic infiltrations and/or bacterial colonies, were present on the prosthesis leaflets in $7.1 \%$ of cases, indicating the presence of inflammation (Figure 9). It is possible that the inflammation of the BP leaflets was secondary to the structural dysfunction or it may have preceded the degenerative changes of the xenomaterial; therefore, these patients were excluded from further analysis.

Indicators of calcium-phosphorus homeostasis and nonspecific inflammatory markers were assessed in $22(41.5 \%)$ patients with histologically confirmed calcification without infection ( $n=53$ ), while defining the indications for reoperation. These patients were assigned to group 1. Group 2 comprised patients $(n=48)$ with normal BP morphology and function, evaluated by transthoracic echocardiography based on matched pairs at the time of primary surgery (Table 2).

\section{Scanning electron microscopy assays}

Scanning electron microscopy was used to assess the degree of tissue damage. Explanted BP-tissue samples (leaflets) were washed and dehydrated through increasing concentrations of ethanol and critical point drying. The samples were

Table I Types of dysfunction according to histological and electron microscopic studies of explanted bioprostheses

\begin{tabular}{ll}
\hline Type of bioprosthetic dysfunction & $\%,(\mathbf{n})$ \\
\hline Tissue degeneration with xenomaterial calcification & $46.9(53)$ \\
Tissue degeneration without calcium deposits & $12.4($ I4) \\
Prosthetic valve endocarditis & $25.7(29)$ \\
Xenomaterial calcification + infection & $7.1(8)$ \\
Prosthesis thrombosis & $0.9(1)$ \\
Not identified & $7.1(8)$ \\
Total & $100(\mathrm{II})$ \\
\hline
\end{tabular}



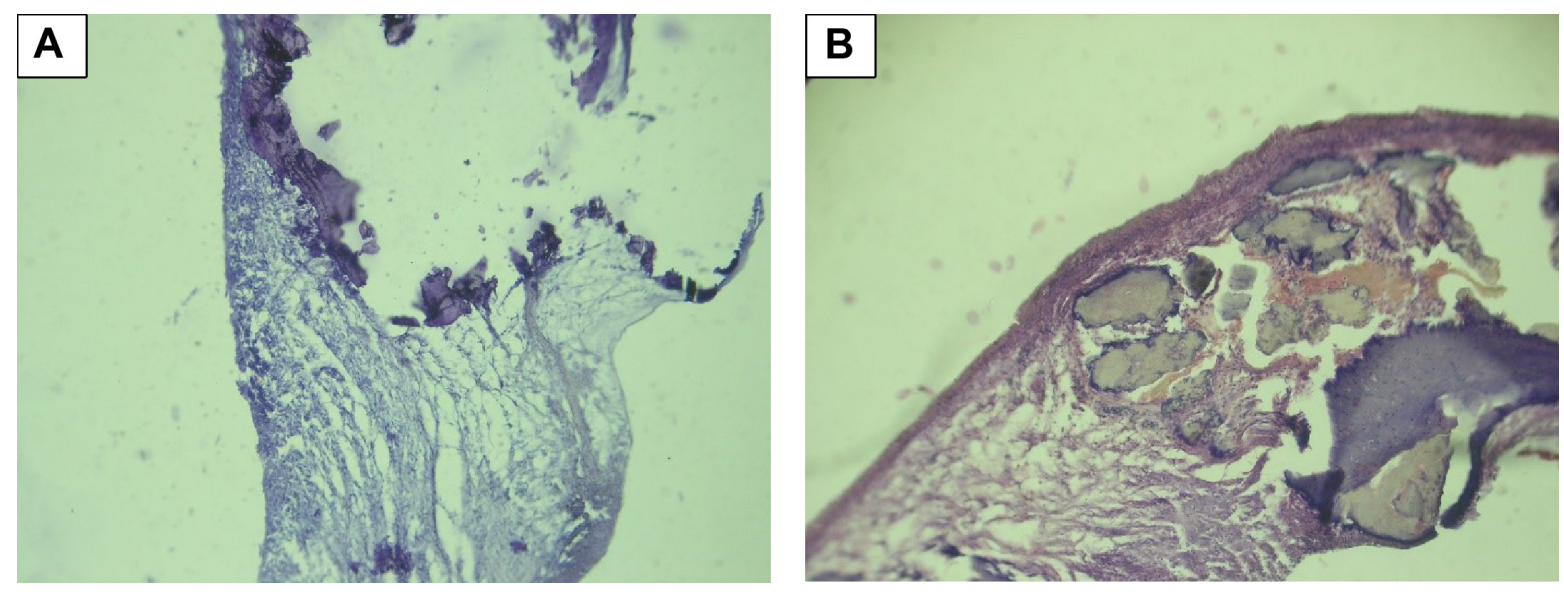

Figure I Macroscopic calcification of bioprosthetic leaflet samples, stained with hematoxylin-eosin, with original magnification $\times 100$. The two figures $(\mathbf{A}$ and $\mathbf{B})$ show the morphology of calcification.

mounted on the specimen stub with double-sided carbon tape and viewed with a TM3000 (Hitachi, Japan) operated under hard vacuum conditions with an accelerating voltage of $15 \mathrm{kV}$ using back-scattered electron and secondary electron modes.

\section{Biochemical assays}

Blood serum was obtained by taking blood from the cubital vein in the morning, on an empty stomach, and transferring it to a Vacuette ${ }^{\circledR} \mathrm{Z}$ serum clot activator (Greiner Bio-One, Austria) blood-collection tubes. Plasma was obtained by taking additional blood into K3EDT Vacuette (Greiner Bio-One) blood-collection tubes. After centrifugation, blood serum was aliquoted and stored at $-40^{\circ} \mathrm{C}$ in a low-temperature refrigerator (MDF; Sanyo, Japan). Blood sampling was performed during scheduled follow-up visits (group 2) or on days 5-7 prior to reoperation necessitated by BP dysfunction (group 1). All procedures were performed with written consent.

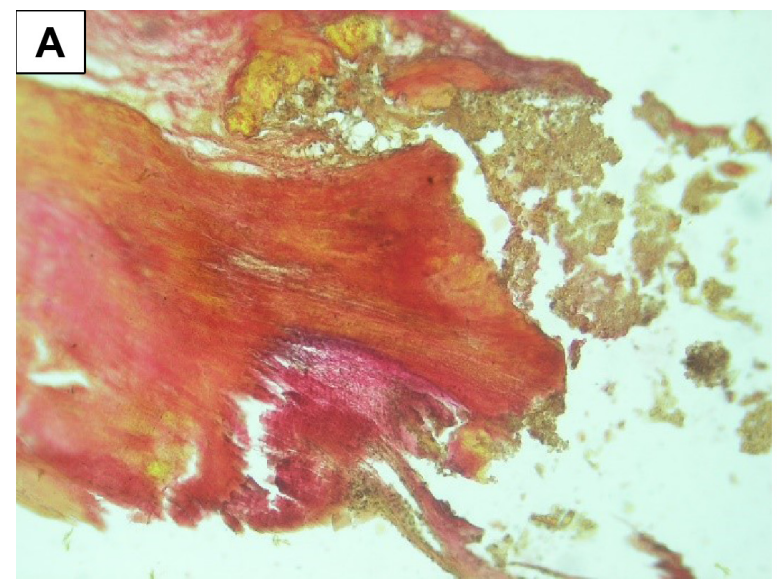

Concentrations of the following analytes were measured using commercially available enzyme-linked immunosorbent assay (ELISA) kits, according to the manufacturers' protocols: parathyroid hormone (PTH; DSL-10-8000 ACTIV 1-PHT; Diagnostic System Laboratories, USA), calcitonin (CV, 2.3\%-4.1\%; Biomerica, USA), 25 -hydroxyvitamin vitamin D $(25[\mathrm{OH}] \mathrm{D}$; Octeia ELISA kit; IDS, UK), osteocalcin (N-Mid ${ }^{\circledR}$; Octeia ELISA kit), bone-specific alkaline phosphatase (Metra BAP; Quidel, USA), osteoprotegerin (OPG; Biomerica), osteopontin (Enzo Life Sciences, USA), IL-1 $\beta,-6,-8,-10$, and -12p40 and TNF $\alpha$ (Bender MedSystems, Austria). The findings were recorded on a Uniplan plate reader (Picone, Russia) using the filters recommended by the manufacturer. Levels of calcium, phosphorus, and alkaline phosphatase were measured using commercial kits (BioSys, Germany) on a Konelab 320i automatic biochemical analyzer (Thermo Fisher Scientific, Finland).

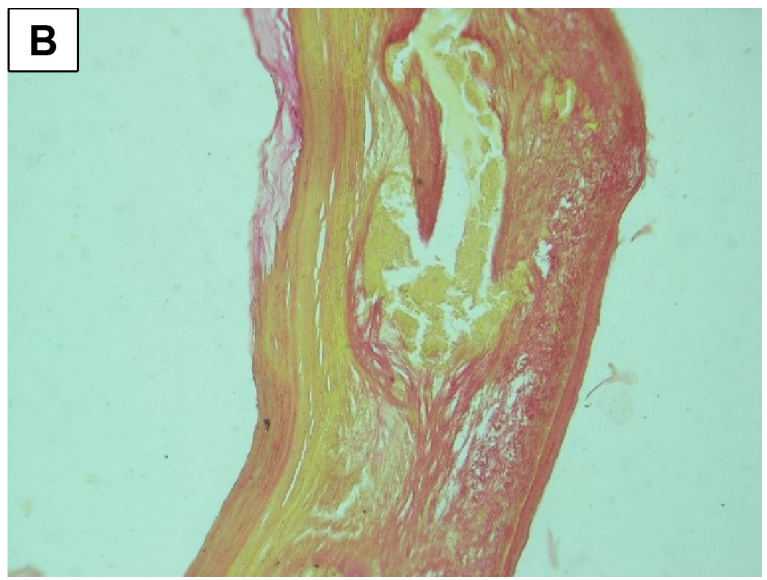

Figure 2 Macroscopic calcification of bioprosthetic leaflet samples, stained with Van Gison, with original magnification $\times 100$. The two panels $(\mathbf{A}$ and B) show the morphology of calcification. 


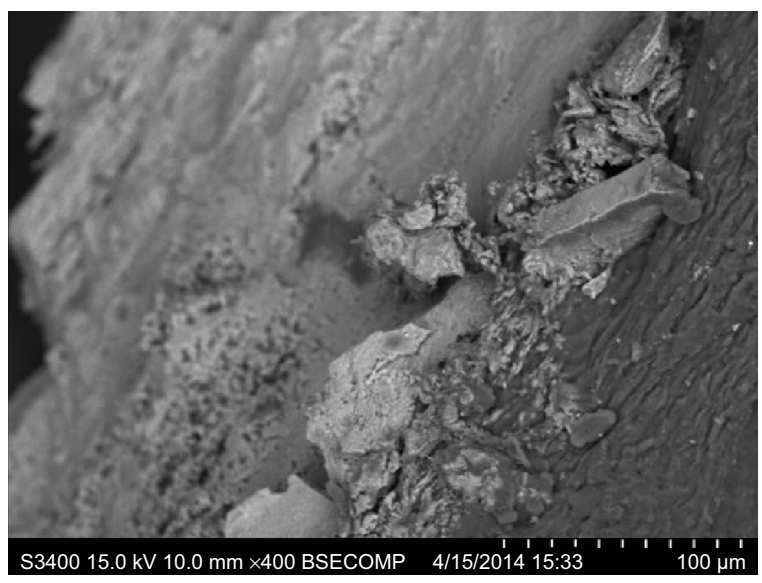

Figure 3 Scanning electron microscopy. Large calcified lumps. Backscatter electron imaging. Scale bar -100 microns.

The parameters with defined reference values, provided by the ELISA manufacturers, were also analyzed in an ageand sex-matched control group of healthy subjects $(n=22)$. This group was formed in order to determine the normal range of the analyzed parameters in the population of the studied geographic region.

\section{Statistical analysis}

Clinical and laboratory data were entered into a Microsoft Excel spreadsheet and analyzed using SAS 6.12, Statistica 6.0, and SPSS software. The Kolmogorov-Smirnov, Lilliefors, and Shapiro-Wilk tests were used to analyze the data from normally distributed data sets. Results are presented as medians and interquartile range. The equality of variances was confirmed using the Cochran-Leuven criterion. Statistical hypotheses were confirmed using nonparametric WhitneyMann-Wilcoxon $U$ and Kruskal-Wallis tests for quantitative

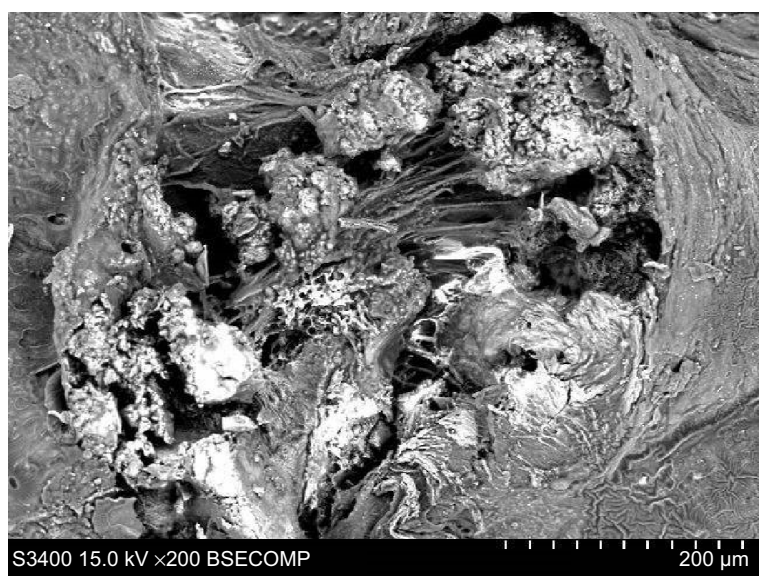

Figure 4 Scanning electron microscopy. Primary tissue degeneration with calcification. Backscatter electron imaging. Scale bar -200 microns.

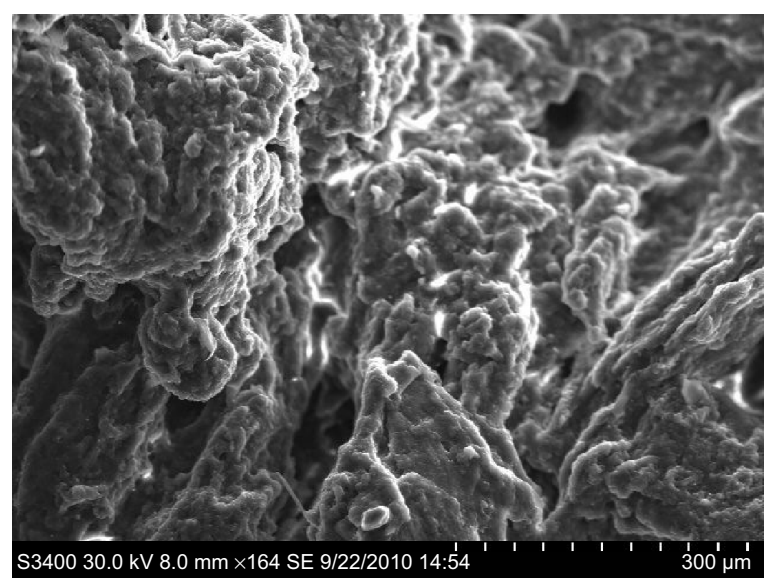

Figure 5 Scanning electron microscopy. Primary tissue degeneration with calcification. Secondary electron imaging. Scale bar -300 microns.

variables. Qualitative traits were assessed using contingency tables with calculation of Pearson's $\chi^{2}$ and odds ratios with $95 \%$ confidence intervals. A $P$-value $<0.05$ was considered statistically significant.

\section{Results}

The groups were comparable in terms of age (group 1, $46.7 \pm 7.5$ years; group 2, $47.9 \pm 5.9$ years; $P>0.05$ ) and sex (group 1, 40.9\% male; group 2, 35.4\% male; $P>0.05$ ) (Table 2). The mean follow-up periods (from the date of primary implantation of BP to reoperation in group 1, from the time of valve replacement to the last follow-up visit in group 2) were 7.2 \pm 3.4 and $8.1 \pm 2.9$ years in groups 1 and 2 , respectively $(P>0.05)$. The predominant etiologic factor for acquired disease was rheumatic heart disease in both groups ( $86 \%$ in group 1 versus $98 \%$ in group $2, P>0.05$ ), while connective tissue dysplasia occurred in $9 \%$ and $2 \%$ of all cases in groups 1 and 2, respectively ( $P>0.05)$, and infective endocarditis was diagnosed in $5 \%$ of patients with calcified valvular BP. There were no significant differences in comorbidities between the groups, though this may have been because of the limited numbers of patients.

Importantly, most patients were in a severe clinical state before primary valve replacement. New York Heart Association functional class IV with chronic heart failure was significantly more prevalent in group 1 compared with group 2 (36\% versus $15 \%, P=0.03$ ). Moreover, patients with calcific degeneration of BP valves had a longer period of CPB during the initial replacement $(112.8 \pm 18.8$ minutes in group 1 versus $97.2 \pm 23.6$ minutes in group $2, P=0.02$ ) (Table 2).

With regard to calcium-phosphorus metabolism, serum total calcium levels remained within the normal range in 

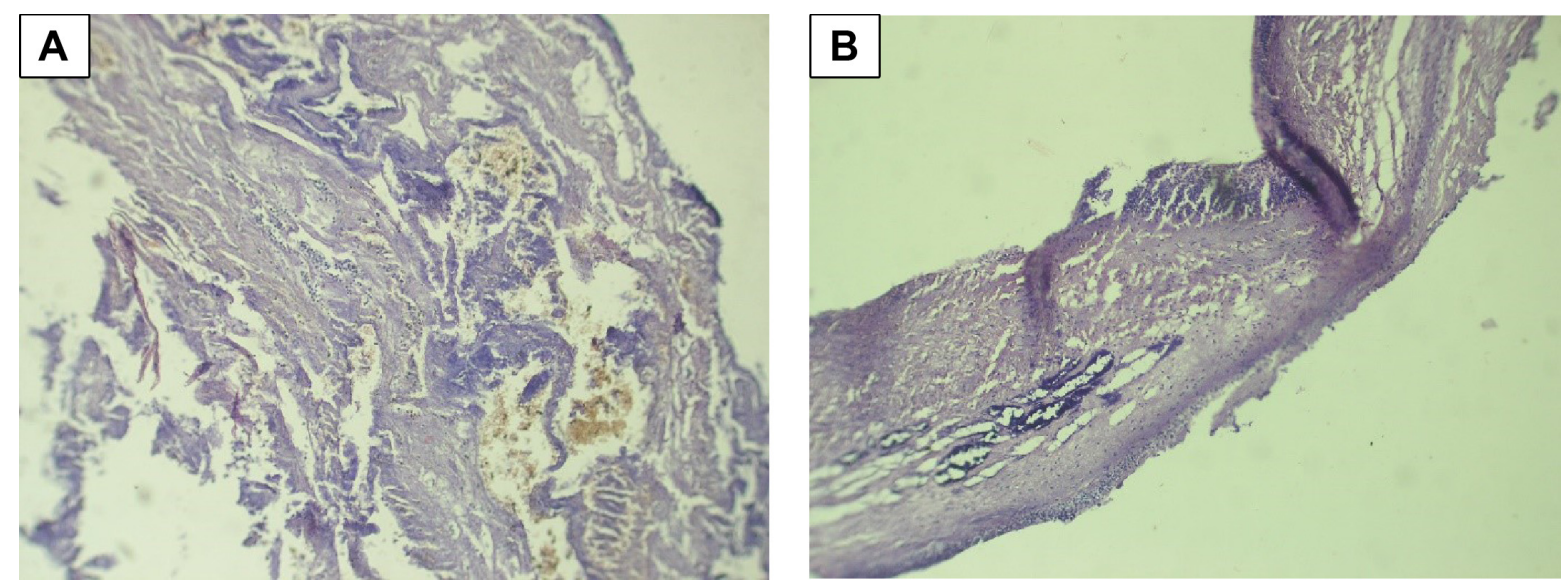

Figure 6 Micro-samples of bioprosthetic leaflet with endocarditis, stained with hematoxylin-eosin. Leuko-, lymphocytic infiltrates are visualized. Original magnification $\times 100$. The two panels (A and $\mathbf{B}$ ) show the morphology of calcification.

patients with confirmed calcification, whereas patients with preserved function of the BP valve had moderate hypercalcemia (2.3 [2.0-2.6] versus $2.6[2.5-2.7] \mathrm{mmol} / \mathrm{L}, P=0.05)$ (Table 3$)$. Serum phosphorus levels were slightly decreased in the group with calcific degeneration $(0.8[0.7-1.3] \mathrm{mmol} / \mathrm{L})$ and corresponded to the lower limit of normal in patients with normal BP function $(0.9[0.8-1.1] \mathrm{mmol} / \mathrm{L})(P>0.05)$. There was no significant difference in calcium:phosphorus ratio between groups 1 and $2(2.4$ [2.0-3.4] versus 2.7 [2.5-3.2], respectively, $P>0.05$ ).

A moderate decrease in serum 25(OH)D levels was reported in both groups, but the deficit was more pronounced in patients with $\mathrm{BP}$ calcification $(34.0$ [21.0-49.4] pmol/L in group 1 versus 40.0 [27.2-54.0] pmol/L in group 2, $P>0.05$ ). A similar tendency was noted in healthy volunteers, where hypovitaminosis D (39.8 [32.2-49.2] pmol/L) was associated with increased parathyroid hormone (PTH) secretion

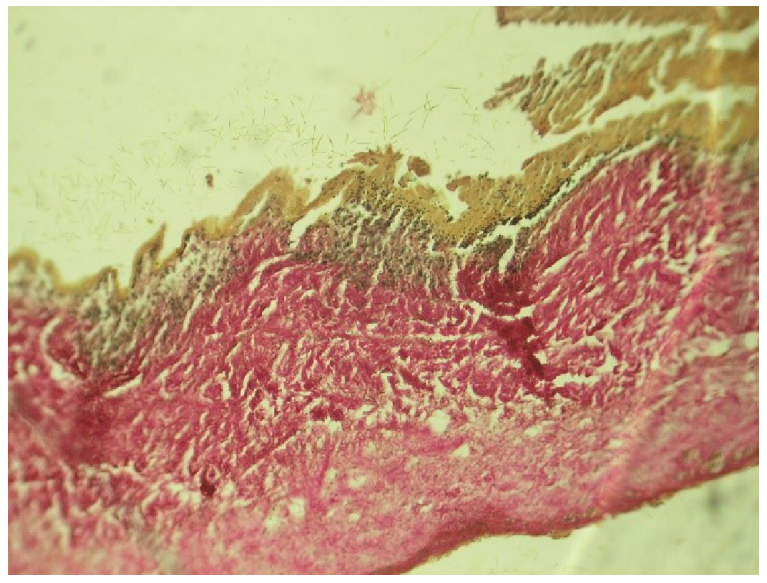

Figure 7 Micro-samples of bioprosthetic leaflet with endocarditis, stained with Van Gison. Original magnification $\times 100$.
(50.4 [36.1-78.7] pg/mL). Despite low levels of 25(OH)D, serum parathyrin levels were within the normal range (37.5 [13.0-70.9] pg/mL in group 1 versus 44.0 [18.2-78.7] pg/mL in group 2).

Serum calcitonin levels remained within the normal reference range in both groups $(7.3[4.7-20.5] \mathrm{pg} / \mathrm{mL}$ in group 1 versus 7.9 [5.2-60.3] pg/mL in group 2, $P>0.05$ ), though levels were slightly increased compared with control levels $(3.8[1.72-5.23] \mathrm{pg} / \mathrm{mL})$.

OPG levels were reduced in the study groups compared with healthy, age-matched subjects (127.6 [95.5-173.0] pg/mL), with the largest deficit in patients with confirmed BP calcification (82.5 [44.2-115.4] $\mathrm{pg} / \mathrm{mL}$ in group 1 versus 113.5 [65.7-191.3] pg/mL in group $2, P>0.05$ ).

Osteocalcin levels in both study groups were within the normal range (10.2 [6.7-23.1] ng/mL versus $12.8[8.3-23.6] \mathrm{ng} / \mathrm{mL}$ in groups 1 and 2, respectively, $(P>0.05)$, but levels

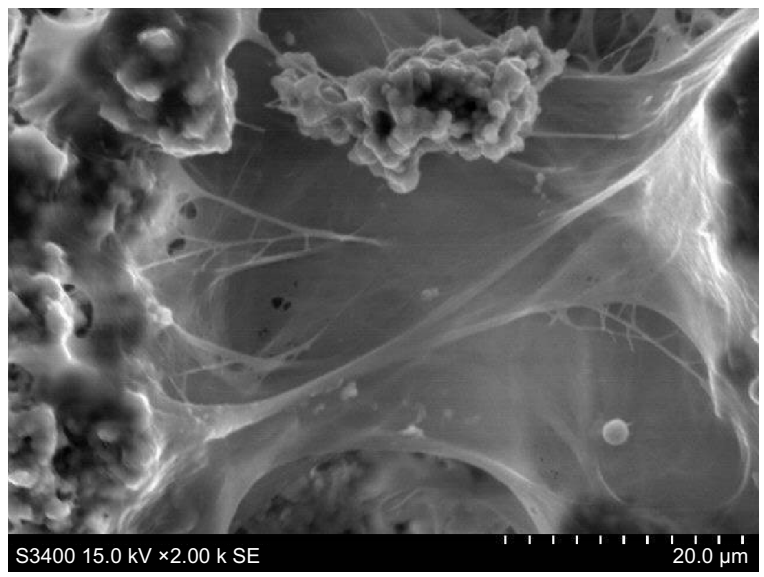

Figure 8 Scanning electron microscopy. Bacteria colonies in fibrin capsules. Secondary electron imaging. Scale bar -200 microns. 


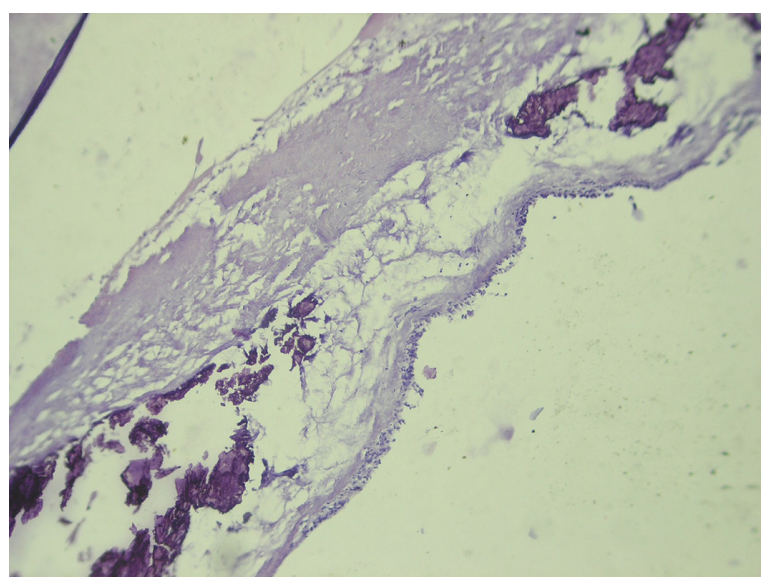

Figure 9 Micro-samples of bioprosthetic leaflet, stained with hematoxylin-eosin. Calcium deposits, leukocytic infiltrates and bacteria colonies are visualized. Original magnification $\times 400$.

were slightly higher in healthy volunteers (17.5 [13.5-20.1] ng/mL).

Osteopontin levels were decreased in both BP groups, regardless of the structural and functional states of the implanted valves $(4.5[3.3-7.7] \mathrm{ng} / \mathrm{mL}$ and $5.2[4.1-7.2] \mathrm{ng} / \mathrm{mL}$ in groups 1 and 2 , respectively; $P>0.05$ ), compared with the control group (17.1 [13.6-21.8] ng/mL).

Serum alkaline phosphatase levels were reduced in patients with preserved BP function (52.0 [42.0-60.0] U/L in group 2 versus $76.5[42.0-90.0] \mathrm{U} / \mathrm{L}$ in group $1, P>0.05$ ), whereas levels of its bone-specific isoenzyme were significantly higher in group 2 compared with patients after reoperation, but remained within the normal range $(22.3$ [15.5-30.5] U/L in group 2 versus 17.1 [12.2-21.4] U/L in group $1, P=0.01)$. It should be noted that the lowest levels of bone-specific alkaline phosphatase were observed in the control group (5.6 [3.3-7.32] U/L).

With regard to the main proinflammatory indicators, serum levels IL-1 $\beta,-6,-10$, and $-12 \mathrm{p} 40$ were increased in BP heart-valve recipients (groups 1 and 2) compared with healthy subjects. However, levels of TNF $\alpha$ were higher in the control group (Table 4).

Patients with calcific BP degeneration (group 1) had slightly higher levels of IL-12p40 (105.6 [93.9-109.8] pg/mL versus 88.8 [76.1-129.5], $P>0.05)$, IL-6 (2.15 [1.94-3.32] pg/mL versus 1.99 [1.91-2.33] $\mathrm{pg} / \mathrm{mL}, P>0.05)$, and IL-10 (3.53 [3.01-4.57] pg/mL versus 2.99 [2.4-3.54] pg/mL, $P>0.05$ ) than those with satisfactory function of the implanted valve (group 2). However, serum IL-8 levels were significantly higher in patients with preserved BP function compared with those with calcification $(13.17[9.72-23.1] \mathrm{pg} / \mathrm{mL}$ in group 2 versus 9.74 [9.19-10.09] pg/mL in group $1, P=0.045)$. IL- $1 \beta$ levels were also slightly higher in group $2(0.78[0.5-1.94] \mathrm{pg} / \mathrm{mL}$ versus 0.55 [0.41-1.62] pg/mL in group $1, P>0.05$ ), though the difference was not significant (Table 4).

Table 2 Clinical data of xenoaortic valve recipients

\begin{tabular}{|c|c|c|c|}
\hline Parameter & $\begin{array}{l}\text { Bioprosthesis } \\
\text { dysfunction (group I) }\end{array}$ & $\begin{array}{l}\text { Preserved bioprosthetic } \\
\text { function (group 2) }\end{array}$ & $\mathbf{P}$ \\
\hline Patients, n (\%) & $22(100)$ & $48(100)$ & \\
\hline Sex, male/female, $n(\%)$ & $9(40.9) / 13(59.1)$ & $17(35.4) / 31(64.6)$ & 0.65 \\
\hline Age, years, mean \pm SD & $46.7 \pm 7.5$ & $47.9 \pm 5.9$ & 0.41 \\
\hline Time from primary surgery, years, mean \pm SD & $7.2 \pm 3.4$ & $8.1 \pm 2.9$ & 0.24 \\
\hline \multicolumn{4}{|l|}{ NYHA FC } \\
\hline FC II, n (\%) & I (5) & $2(4)$ & 0.81 \\
\hline FC III, n (\%) & $14(64)$ & $36(75)$ & 0.37 \\
\hline FC IV, n (\%) & $8(36)$ & $7(15)$ & 0.03 \\
\hline \multicolumn{4}{|l|}{ Etiology of disease } \\
\hline RHD, n (\%) & $19(86)$ & $47(98)$ & 0.06 \\
\hline IE, n (\%) & I (5) & - & - \\
\hline CCTD, n (\%) & $2(9)$ & I (2) & 0.19 \\
\hline \multicolumn{4}{|l|}{ Comorbidities } \\
\hline CAD, n (\%) & $2(9)$ & $6(13)$ & 0.67 \\
\hline DM, n (\%) & I (5) & $2(4)$ & 0.70 \\
\hline Arterial hypertension, n (\%) & $6(27)$ & $20(42)$ & 0.24 \\
\hline Polyvalvular disease, $\mathrm{n}(\%)$ & $2(9)$ & - & - \\
\hline ACVA, n (\%) & $2(5)$ & II (23) & 0.17 \\
\hline \multicolumn{4}{|l|}{ Intraoperative parameters } \\
\hline CPB time, minutes & $112.8 \pm 18.8$ & $97.2 \pm 23.6$ & 0.02 \\
\hline Ischemia time, minutes & $82.3 \pm 14.2$ & $82.6 \pm 15.8$ & 0.51 \\
\hline
\end{tabular}

Abbreviations: SD, standard deviation; NYHA, New York Heart Association; FC, functional class; RHD, rheumatic heart disease; IE, infective endocarditis; CCTD, cardiac connective tissue dysplasia; $\mathrm{AH}$, arterial hypertension; $\mathrm{CAD}$, coronary artery disease; $\mathrm{DM}$, diabetes mellitus; $\mathrm{ACVA}$, acquired valvular heart disease; CPB, cardiopulmonary bypass. 
Table 3 Indicators of calcium-phosphorus metabolism in xenoaortic valve recipients

\begin{tabular}{|c|c|c|c|c|c|}
\hline Indicator & $\begin{array}{l}\text { Normal } \\
\text { range }\end{array}$ & $\begin{array}{l}\text { Control group } \\
(n=22)\end{array}$ & $\begin{array}{l}\text { Bioprosthetic dysfunction } \\
\text { (group I, } n=22 \text { ) }\end{array}$ & $\begin{array}{l}\text { Preserved bioprosthetic } \\
\text { function (group 2, } n=48 \text { ) }\end{array}$ & $P^{*}$ \\
\hline Calcium, mmol/L & $2.1-2.5$ & - & $2.3(2.0 ; 2.6)$ & $2.6(2.5 ; 2.7)$ & 0.05 \\
\hline Phosphorus, mg/dL & $0.9-1.9$ & - & $0.8(0.7 ; 1.3)$ & $0.9(0.8 ; 1.1)$ & 0.43 \\
\hline Calcium/phosphorus & - & - & $2.4(2.0 ; 3.4)$ & $2.7(2.5 ; 3.2)$ & 0.30 \\
\hline Vitamin D, pmol/L & $47.7-144^{\dagger}$ & $39.8(32.2 ; 49.2)$ & $34.0(21.0 ; 49.4)$ & $40.0(27.2 ; 54.0)$ & 0.36 \\
\hline PTH, pg/mL & $21-45^{\dagger}$ & $50.4(36.1 ; 78.7)$ & $37.5(13.0 ; 70.9)$ & $44.0(18.2 ; 78.7)$ & 0.32 \\
\hline Calcitonin, $\mathrm{pg} / \mathrm{mL}$ & $<30 *$ & $3.8(1.72 ; 5.23)$ & $7.3(4.7 ; 20.5)$ & $7.9(5.2 ; 60.3)$ & 0.34 \\
\hline OPG, pg/mL & - & $127.6(95.5 ; 173.0)$ & $82.5(44.2 ; 115.4)$ & II $3.5(65.7 ; 191.3)$ & 0.32 \\
\hline Osteocalcin, ng/mL & $9.6-40 *$ & $17.5(13.5 ; 20.1)$ & $10.2(6.7 ; 23.1)$ & $12.8(8.3 ; 23.6)$ & 0.33 \\
\hline Osteopontin, ng/mL & - & I7.I (I3.6;2I.8) & $4.5(3.3 ; 7.7)$ & $5.2(4.1 ; 7.2)$ & 0.57 \\
\hline ALP, U/L & $70-306$ & - & $76.5(42.0 ; 90.0)$ & $52.0(42.0 ; 60.0)$ & 0.09 \\
\hline Bone isoenzyme of ALP, U/L & $15-41.3 *$ & $5.6(3.3 ; 7.32)$ & I7.I (I2.2;2I.4) & $22.3(15.5 ; 30.5)$ & 0.01 \\
\hline
\end{tabular}

Notes: *Group I versus group 2; †reference values provided by the manufacturer of each laboratory kit. Values given as median (interquartile range).

Abbreviations: PTH, parathyroid hormone; OPG, osteoprotegerin; ALP, alkaline phosphatase.

We investigated the association between subclinical inflammation and disorders of calcium-phosphorus homeostasis in recipients of BP heart valves and detected significant inverse relationships between serum IL-10 and vitamin D $(r=-0.355, P<0.05)$, and between IL-6 and calcitonin $(r=-0.423, P<0.05)$. There were also significant direct correlations between serum IL-12p40 and osteopontin ( $r=0.397$, $P<0.05)$, and between IL-6 and alkaline phosphatase $(r=0.507, P<0.05)$ (Table 5).

\section{Discussion}

The rate of xenogeneic tissue calcification of BP heart valves depends on factors related to the implanted BP device, the transformation of biomaterials during preservation and subsequent function of the prosthesis, and recipient-related factors. ${ }^{1-3}$ In addition to age, the current medical literature includes dyslipidemia, smoking, and concomitant diseases, such as diabetes mellitus and metabolic syndrome, as risk factors for calcification. ${ }^{11-13,15}$ The negative impact of these proatherogenic factors on BP functioning occurs via the activation of nonspecific inflammatory responses. ${ }^{7-10}$ Moreover, complex biological and metabolic relationships exist between atherosclerotic lesions and bone mineral density, ${ }^{7-9,16-21}$ through the expression of proinflammatory markers and regulatory proteins, which control osteosynthesis and bone resorption..$^{20-24}$ The pathological mineralization of soft tissues may thus involve complex underlying mechanisms. ${ }^{9,10}$ We therefore retrospectively compared calcium-phosphorus metabolism and proinflammatory status in patients with preserved BP heart-valve function and those with calcific degeneration.

To avoid the possible influence of device characteristics on the rate of structural dysfunction, only subjects who received identical BP types were included in the study (groups 1 and 2), at comparable times from primary surgery. The potential impacts of central hemodynamics on the structural and functional states of the BP were minimized by selecting devices implanted in the mitral position. There were no significant differences in age or etiological factors between the studied groups of patients (Table 2).

Patients with long-term calcific BP degeneration (group 1) had a more severe clinical state prior to mitral valve replacement compared with those with intact morphologic and functional implanted BPs (group 2). In addition, patients with $\mathrm{BP}$ dysfunction had a longer $\mathrm{CPB}$ period during primary surgery. These results suggest that the initial clinical severity

Table 4 Nonspecific inflammatory markers in xenoaortic valves

\begin{tabular}{|c|c|c|c|c|}
\hline Indicator & $\begin{array}{l}\text { Control group } \\
(n=22)\end{array}$ & $\begin{array}{l}\text { Bioprosthetic dysfunction } \\
\text { (group I, } n=22 \text { ) }\end{array}$ & $\begin{array}{l}\text { Preserved bioprosthetic } \\
\text { function (group 2, } n=48 \text { ) }\end{array}$ & P* \\
\hline $\mathrm{IL}-\mathrm{I} \beta, \mathrm{pg} / \mathrm{mL}$ & $0.39(0.28 ; 1.68)$ & $0.55(0.4|;| .62)$ & $0.78(0.5 ; 1.94)$ & 0.37 \\
\hline $\mathrm{IL}-6, \mathrm{pg} / \mathrm{mL}$ & $0.57(0.01 ; 3.02)$ & $2.15(1.94 ; 3.32)$ & $1.99(1.91 ; 2.33)$ & 0.31 \\
\hline IL-8, pg/mL & $12.88(5.01 ; \mid 15.66)$ & $9.74(9.19 ; 10.09)$ & $13.17(9.72 ; 23.1)$ & 0.045 \\
\hline $\mathrm{IL}-10, \mathrm{pg} / \mathrm{mL}$ & $1.05(0.25 ; 2.24)$ & $3.53(3.01 ; 4.57)$ & $2.99(2.4 ; 3.54)$ & 0.29 \\
\hline IL-I2p40 & $24.9(12.9 ; 53.9)$ & $105.6(93.9 ; 109.8)$ & $88.8(76.1 ; 129.5)$ & 0.44 \\
\hline $\mathrm{TNF} \alpha$ & $1.58(1.00 ; 4.39)$ & $0.01(0.01 ; 0.49)$ & $0.08(0.01 ; 0.8)$ & 0.53 \\
\hline
\end{tabular}

Notes: *Group I versus group 2. Values given as median (interquartile range). 
Table 5 Correlations between calcium-phosphorus metabolism and nonspecific inflammatory markers

\begin{tabular}{lllllll}
\hline & IL-1 2p40 & IL-10 & IL-8 & IL-6 & IL-I $\beta$ & TNF $\alpha$ \\
\hline Calcium, mmol/L & -0.170 & 0.045 & 0.173 & -0.046 & 0.099 & 0.085 \\
Phosphorus, mg/dL & -0.066 & -0.104 & -0.002 & -0.127 & 0.237 & -0.114 \\
Calcium/phosphorus & -0.094 & $-0.355^{*}$ & 0.258 & -0.308 & -0.050 & -0.164 \\
Vitamin D, pmol/L & 0.031 & -0.131 & -0.028 & 0.234 & -0.126 & -0.111 \\
PTH, pg/mL & -0.190 & 0.279 & -0.101 & $-0.423^{*}$ & -0.024 & -0.114 \\
Calcitonin, pg/mL & 0.050 & 0.242 & 0.253 & 0.048 & 0.287 & 0.27 I \\
OPG, pg/mL & 0.027 & 0.010 & 0.139 & 0.171 & -0.0427 & -0.054 \\
Osteocalcin, ng/mL & $0.397^{*}$ & 0.103 & 0.035 & -0.077 & -0.077 & -0.124 \\
Osteopontin, ng/mL & -0.173 & -0.045 & 0.142 & $0.507 *$ & 0.0827 & 0.199 \\
ALP, U/L & 0.165 & -0.037 & 0.264 & -0.048 & $-0.04 I$ & -0.192 \\
\hline
\end{tabular}

Note: $* p<0.05$.

Abbreviations: PTH, parathyroid hormone; OPG, osteoprotegerin; ALP, alkaline phosphatase.

of the patient, determined by the degree of preoperative decompensated chronic heart failure and the severity of the postoperative systemic inflammatory response and homeostatic disturbances, may affect the life span of the BP.

We assessed calcium-phosphorus metabolism in the two groups of patients. Calcium and phosphorus homeostasis is part of a multifactorial, tightly controlled system, which functions to maintain serum calcium and phosphorus levels within their physiological ranges. Variations in components of this regulatory system might therefore be expected to potentiate the pathological mineralization of soft tissue and the calcific degeneration of BPs.

Serum levels of calcium and inorganic phosphate are known to depend on the function of the parathyroid gland, vitamin $\mathrm{D}$ activity, and specific characteristics of bone metabolism associated with these factors. The current analysis found that patients with preserved BP function (group 2) had moderate hypercalcemia compared with those with calcific degeneration (group 1), who had normal serum calcium levels.

Serum calcium is directly affected by PTH, which functions to prevent hypocalcemia, enhance bone resorption, stimulate renal calcium reabsorption, and hydroxylate vitamin D. Vitamin D deficiency thus promotes hypersecretion of PTH. This relationship was only found in the control group in the present study, whereas serum PTH levels in BP recipients (groups 1 and 2) were within the normal range, despite moderate hypovitaminosis $\mathrm{D}$, probably because of the absence of hypocalcemia in these patients.

Despite extensive studies, the biological role of vitamin D remains unclear. It is known to antagonize PTH, and its deficiency stimulates the expression of proinflammatory factors, including cytokines, metalloproteinases, and C-reactive protein. ${ }^{25,26}$ In addition to estrogen and gastrointestinal peptides, vitamin $\mathrm{D}$ is also known to regulate the secretion of calcitonin, which is an indirect antagonist of PTH produced by thyroid cells.

The main effect of calcitonin is to reduce serum calcium levels via inhibition of osteoclast activity. ${ }^{27}$ Hypercalcemia stimulates the secretion of calcitonin, thus promoting the deposition of excess calcium in bones, and increased levels of calcitonin have thus been suggested to provoke the calcification of native heart valves. In the present study, serum calcitonin levels were significantly higher in the study groups compared with the control group, though the values remained within the normal reference ranges. We were therefore unable to demonstrate any impact of calcitonin on BP calcification, but further studies of its biological role are warranted.

OPG is a key inhibitor of osteoclast differentiation, and prevents bone resorption. ${ }^{26} \mathrm{OPG}$ deficiency is known to lead to severe osteoporosis, associated with abnormal calcification of soft tissues..$^{28,29}$ The present study showed that OPG levels were decreased in both study groups compared with healthy subjects, with a more pronounced decrease in patients with confirmed calcification. The mechanism responsible for this phenomenon is unclear, but it may be associated with disorders of bone remodeling, which are the underlying cause of many pathological processes, including mineralization of cardiovascular system elements and calcific degeneration of BPs.

Biochemical components of bone tissue, such as matrix proteins (osteocalcin, osteopontin, etc) and alkaline phosphatase enzymes, as potential markers of disorders of calciumphosphorus metabolism, represent a promising area for the study of abnormal calcification of biological structures. ${ }^{30,31}$ One of the most sensitive indicators of bone metabolism, osteocalcin, showed similar levels in patients with preserved function (group 2) and those with calcific degeneration of 
implanted prosthetic valves (group 1). However, relatively higher levels in the control group may provide indirect evidence of differences in calcium-phosphorus homeostasis between healthy subjects and BP heart-valve recipients.

The noncollagenous protein osteopontin is known to affect the synthetic functions, migration, and differentiation of pro-osteogenic cell lines, and to regulate the physiological processes of bone formation and pathological tissue mineralization. ${ }^{32,33}$ Research has shown that osteopontin plays a pivotal role in the pathogenesis of calcific aortic valve stenosis. ${ }^{34}$ Osteopontin may exacerbate local inflammation by regulating the activity of cytokines and metalloproteinases. However, clinical and experimental research demonstrated that osteopontin may inhibit the pathological calcification process by suppressing mineral deposition and/ or active resorption. ${ }^{35}$ The most likely protective mechanisms of osteopontin involve self-aggregation and adhesion of hydroxyapatite crystals on the surface, through binding to specific residual amino acids. ${ }^{36}$ The more pronounced decrease in osteopontin levels in patients with degenerative BP changes demonstrated in the present study may have been caused by either increased consumption (by binding to mineral deposits in the prosthetic leaflet) or by depletion of its products under clinically severe calcification.

Alkaline phosphatase is involved in the metabolism of phosphoric acid and contributes to phosphorus transport in the body, though details of its mechanism have not been well studied. Serum levels of bone-specific alkaline phosphatase are known to reflect the metabolic state of osteoblasts, the cells responsible for bone-tissue formation. Osteoclasts, which are involved in bone resorption, are also capable of releasing alkaline phosphatase, and a moderate increase in bone-specific alkaline phosphatase is associated with osteolytic activity. ${ }^{26}$ The reduction in the bone isoenzyme of alkaline phosphatase in patients with calcific BP degeneration in the present study may be associated with reduced osteosynthetic ability of osteoblasts during the final stage of calcific BP degeneration.

In this study, patients with symptomatic BP dysfunction and moderate hypovitaminosis D, OPG, and osteopontin deficiencies, but with normal PTH and total calcium levels had significantly reduced serum levels of bone-specific alkaline phosphatase isoenzymes, compared with recipients with well-functioning BPs.

This study also assessed the main proinflammatory indicators in the study groups. The current medical literature reports an association between systemic inflammation and the severity of coronary atherosclerosis. ${ }^{37} \mathrm{We}$ supposed that the activity of some nonspecific markers of the inflammatory response may reflect the severity of the clinical state of the patient, as well as acting as prognostic factors for the early development of calcific BP degeneration.

The elevated levels of proinflammatory IL-1 $\beta$, IL- 6 , and IL-12p40 in both groups in the present study compared with healthy subjects were likely associated with the underlying disease and the patient's response to the implanted prosthetic device. The high levels of anti-inflammatory IL-10 may reflect a compensatory reaction, while the reduced TNF $\alpha$ levels seen in both groups of patients may be explained by the insolvency of the inflammatory response.

The elevated levels of IL-12p40, IL-6, and IL-10 in patients with calcific degeneration (group 1) compared with the group with preserved BP function (group 2) reflect the severity of the depression of intracardiac and systemic hemodynamics, associated with clinically significant dysfunction. However, the significant increase in serum IL-8 in patients with preserved BP function (group 2) was particularly notable. IL-8 is an inducible protein produced following the effect of mitogens or endogenous regulators on immune cells. IL-8 is a chemokine that regulates the chemotaxis of various cell types (neutrophils, monocytes, eosinophils, and T-cells) in the inflammatory area. Moreover, IL-8 regulates neutrophil adhesion by modifying the expression of integrins and other compounds with adhesive properties. The ability of IL-8 to cause the migration of cells and stimulate their adhesion makes it an active participant in the inflammatory response.

Our results are consistent with other studies, which showed a high prognostic significance of elevated IL-6 and decreased IL-8 levels in terms of the identification of significant coronary artery calcification in patients with atypical chest pain. ${ }^{37}$ Therefore, low serum levels of IL- 8 may have a negative impact on the rate of pathological mineralization of soft tissue in atherosclerotic vascular lesions, as well as in the calcification of BP leaflets. Larger studies of BP heart-valve recipients with long-term follow-up are required to confirm this hypothesis.

Any implanted foreign biological material causes an immune response. Several experimental studies have demonstrated the infiltration of immune cells (macrophages, lymphocytes, polymorphonuclear leukocytes, etc) with cytokine-producing activity in explanted xenopericardial samples. ${ }^{38}$ Migrating cells may produce proteolytic enzymes (metalloproteinases, cathepsins), known as stimulants of osteoblastic differentiation of myofibroblasts and smoothmuscle cells, ${ }^{10}$ which exert pro-osteogenic activity and promote the formation of micro- and macrocalcifications, 
destroying the intercellular matrix and collagen fibers. We therefore investigated the association between subclinical inflammation and disorders of calcium-phosphorus homeostasis in BP heart-valve recipients, and demonstrated significant relationships between serum levels of IL-10, IL-6, and IL-12p40 and vitamin D, calcitonin, alkaline phosphatase, and osteopontin, thus confirming an association between subclinical inflammation and mineral metabolism.

The detailed interpretation of the results, identification of the relationships, and formulation of the conclusions should take into consideration the following limitations. This was a retrospective study, with a small number of patients in both groups. We did not monitor the parameters of mineral metabolism and inflammatory markers over a long period (from the time of primary surgery to the development of clinically significant BP dysfunction). Furthermore, there was a lack of information on bone mineral density in the study groups, and a lack of information regarding regular medical therapy (including hormone-replacement therapy). However, despite these limitations, the present study confirmed the existence of active, cell-mediated regulation of abnormal calcification of soft tissues, suggesting that abnormalities of calcium-phosphorus metabolism and the severity of the nonspecific inflammatory response are contributory factors affecting the life span of BPs.

\section{Conclusion}

The results of this study suggest that recipient metabolic status, defined by the active processes of bone resorption and local and systemic inflammation, should be considered possible predictors determining the rate of structural dysfunction of BPs. We also suggest that calcification degeneration of valvular BPs is a multistage process, manifested by subsequent activation of different regulatory levels.

Further studies are required to confirm this hypothesis, including an assessment of recipient bone mineral density, a more thorough study of recipient immunologic status, and the identification of possible genetic determinants of pathological calcification. The results of the current and future studies will allow the pathogenic mechanisms and risks of BP dysfunction and pathological calcification to be defined.

\section{Author contributions}

$\mathrm{NR}, \mathrm{OB}$ and LB were a principal investigators, study coordinator, and investigator, participated in all stages of recruitment of the patients and in analysis of the data, and drafted and critically reviewed the manuscript. $\mathrm{OH}, \mathrm{AP}$, and
EU were study coordinators and investigators, participated in all stages of recruitment of patients and in analysis of the data, and drafted and critically reviewed the manuscript. NK and YO were study investigators, participated in all stages of recruitment of patients, and critically reviewed the manuscript. OB and LS were principal investigators. All other study investigators conducted the study and collected the data. All authors contributed toward data analysis, drafting and revising the paper, approved the final version, and agree to be accountable for all aspects of the work.

\section{Disclosure}

The authors report no conflicts of interest in this work.

\section{References}

1. Tillquist M, Maddox T. Cardiac crossroads: deciding between mechanical or bioprosthetic heart valve replacement. Patient Prefer Adherence. 2011;5:91-99.

2. Pibarot P, Dumesnil J. Prosthetic heart valves: selection of the optimal prosthesis and long-term management. Circulation. 2009;119(7): 1034-1048.

3. Oakley R, Kleine P, Bach DS. Choice of prosthetic heart valve in today's practice. Circulation. 2008;117(2):253-256.

4. Bonow R, Carabello B, Kanu C, et al. ACC/AHA 2006 guidelines for the management of patients with valvular heart disease: a report of the American College of Cardiology/American Heart Association Task Force on Practice Guidelines (writing committee to revise the 1998 Guidelines for the Management of Patients With Valvular Heart Disease): developed in collaboration with the Society of Cardiovascular Anesthesiologists: endorsed by the Society for Cardiovascular Angiography and Interventions and the Society of Thoracic Surgeons. Circulation. 2006;114(5):84-231.

5. Vesey JM, Otto CM. Complication of prosthetic heart valves. Curr Cardiol Rep. 2004;6(2):106-111.

6. Thiene G, Valente M. Anticalcification strategies to increase bioprosthetic valve durability. J Heart Valve Dis. 2011;20(1):37-44.

7. Aronow WS. Osteoporosis, osteopenia, and atherosclerotic vascular disease. Arch Med Sci. 2011;7(1):21-26.

8. Miller JD, Weiss RM, Heistad DD. Calcific aortic valve stenosis: methods, models, and mechanisms. Circ Res. 2011;108(11):1392-1312.

9. Hjortnaes J, Butcher J, Figueiredo JL, et al. Arterial and aortic valve calcification inversely correlates with osteoporotic bone remodelling: a role for inflammation. Eur Heart J. 2010;31(16):1975-1984.

10. New SE, Aikawa E. Molecular imaging insights into early inflammatory stages of arterial and aortic valve calcification. Circ Res. 2011;108(11): 1381-1391.

11. Mahjoub H, Mathieu P, Sénéchal M, et al. ApoB/ApoA ratio is associated with increased risk bioprosthetic valve degeneration. $\mathrm{J} \mathrm{Am}$ Coll Cardiol. 2013;61(7):752-761.

12. Shetty R, Pibarot P, Auget A, et al. Lipid-mediated inflammation and degeneration of bioprosthetic heart valves. Eur J Clin Invest. 2009;39(6): 471-480.

13. Briand M, Pibarot P, Després JP, et al. Metabolic syndrome is associated with faster degeneration of bioprosthetic valves. Circulation. 2006; 114(1):1512-1517.

14. Schoen FJ, Levy RJ. Calcification of tissue heart valve substitutes: progress toward understanding and prevention. Ann Thorac Surg. 2005; 79(3):1072-1080.

15. Ruel M, Kulik A, Rubens FD, et al. Late incidence and determinants of reoperation in patients with prosthetic heart valves. Eur J Cardiothorac Surg. 2004;25(3):364-370. 
16. Kiel DP, Kauppila LI, Cupples LA, Hannan MT, O’Donnell CJ, Wilson PW. Bone loss and the progression of abdominal aortic calcification over a 25 year period: the Framingham Heart Study. Calcif Tissue Int. 2001;68(5):271-276.

17. Tankò LB, Bagger YZ, Christiansen C. Low bone mineral density in the hip as a marker of advanced atherosclerosis in elderly women. Calcif Tissue Int. 2003;73(1):15-20.

18. Yamaguchi T, Sugimoto T, Yano S, et al. Plasma lipids and osteoporosis in postmenopausal women. Endocr J. 2002;49(2):211-217.

19. Hak AE, Pols HA, van Hemert AM, Hofman A, Witteman JC. Progression of aortic calcification is associated with metacarpal bone loss during menopause: a population-based longitudinal study. Arterioscler Thromb Vasc Biol. 2000;20(8):1926-1931.

20. Abedin M, Tintut Y, Demer LL. Vascular calcification. Mechanisms and clinical ramifications. Arterioscler Thromb Vasc Biol. 2004;24(7): 1161-1170.

21. Doherty TM, Fitzpatrick LA, Inoue F, et al. Molecular, endocrine, and genetic mechanisms of arterial calcification. Endocr Rev. 2004;25(4): 629-672.

22. Rubin MR, Silverberg SJ. Vascular calcification and osteoporosis - the nature of the nexus. J Clin Endocrinol Metab. 2004;89(9):4243-4245.

23. Dhore CR, Cleutjens JP, Lutgens E, et al. Differential expression of bone matrix regulatory proteins in human atherosclerotic plaques. Arterioscler Thromb Vasc Biol. 2001;21(12):1998-2003.

24. Vattikuti R, Towler DA. Osteogenic regulation of vascular calcification: an early perspective. Am J Physiol Endocrinol Metab. 2004; 286(5):686-696.

25. Zitterman A. Vitamin D in preventive medicine: are we ignoring the evidence? Br J Nutr. 2003;89(5):552-572.

26. Hamerman D. Osteoporosis and atherosclerosis: biological linkages and the emergence of dual-purpose therapies. QJM. 2005;98(7):467-484.

27. Conway S. Osteoporosis in cystic fibrosis. J Cyst Fibros. 2003;2(4): 161-162.

28. Nakamura M, Udagawa N, Matsuura S, et al. Osteoprotegerin regulates bone formation through a coupling mechanism with bone resorption. Endocrinology. 2003;144(12):5441-5449.
29. Min H, Morony S, Sarosi I, et al. Osteoprotegerin reverses osteoporosis by inhibiting endosteal osteoclasts and prevents vascular calcification by blocking a process resembling osteoclastogenesis. J Exp Med. 2000;192(4):463-474.

30. Pettenazzo E, Deiwick M, Thiene G, et al. Dynamic in vitro calcification of bioprosthetic porcine valves: evidence of apatite crystallization. J Thorac Cardiovasc Surg. 2004;21(3):500-509.

31. Sacks MS, Schoen FJ. Collagen fiber disruption occurs independent of calcification in clinically explanted bioprosthetic heart valves. J Biomed Mater Res. 2002;62(3):359-371.

32. Srivatsa SS, Harrity PJ, Maercklein PB, et al. Increased cellular expression of matrix proteins that regulate mineralization is associated with calcification of native human and porcine xenograft bioprosthetic heart valves. J Clin Invest. 1997;99(5):996-1009.

33. Wada T, McKee MD, Steitz S, Giachelli CM. Calcification of vascular smooth muscle cell cultures: inhibition by osteopontin. Circ Res. 1999; 84(2):166-178.

34. Yu PJ, Skolnick A, Ferrari G, et al. Correlation between plasma osteopontin levels and aortic valve calcification: potential insights into the pathogenesis of aortic valve calcification and stenosis. J Thorac Cardiovasc Surg. 2009;138(1):196-199.

35. Steitz SA, Speer MY, McKee MD, et al. Osteopontin inhibits mineral deposition and promotes regression of ectopic calcification. Am J Pathol. 2002;161(6):2035-2046.

36. Goldsmith HL, Labrosse JM, McIntosh FA, Mäenpää PH, Kaartinen MT, McKee MD. Homotypic interactions of soluble and immobilized osteopontin. Ann Biomed Eng. 2002;30(6):840-850.

37. Raaz-Schrauder D, Klinghammer L, Baum C, et al. Association of systemic inflammation markers with the presence and extent of coronary artery calcification. Cytokine. 2012;57(2):251-257.

38. Thampi P, Nair D, Lalithakunjamma R, Vijayan N, Venugopal S, Ramachandra U. Pathological effects of processed bovine pericardial scaffolds - a comparative in vivo evaluation. Artif Organs. 2013 37(7):600-605.
Patient Preference and Adherence

\section{Publish your work in this journal}

Patient Preference and Adherence is an international, peer-reviewed, open access journal that focuses on the growing importance of patient preference and adherence throughout the therapeutic continuum. Patient satisfaction, acceptability, quality of life, compliance, persistence and their role in developing new therapeutic modalities and compounds to optimize

\section{Dovepress}

clinical outcomes for existing disease states are major areas of interest for the journal. This journal has been accepted for indexing on PubMed Central. The manuscript management system is completely online and includes a very quick and fair peer-review system, which is all easy to use. Visit http://www. dovepress.com/testimonials.php to read real quotes from published authors. 